\title{
HIGHLY STABLE SURFACE FUNCTIONALIZATION OF HIGH-ASPECT-RATIO MICROGAS CHROMATOGRAPHY COLUMNS USING LAYER-BY-LAYER SELF-ASSEMBLY OF $\mathrm{SiO}_{2}$ NANOPARTICLES Dong Wang $^{l+}$, Hamza Shakeel ${ }^{2 \dagger}$, Reza Montazami ${ }^{3}$, Gary Rice ${ }^{4}$, James R. Heflin ${ }^{l}$, and Masoud Agah ${ }^{2 *}$ ${ }^{1}$ Department of Physics, Virginia Tech, USA \\ ${ }^{2}$ VT MEMS Lab, Bradley Department of Electrical \& Computer Engineering, Virginia Tech, USA \\ ${ }^{3}$ Department of Mechanical Engineering, Iowa State University, USA \\ ${ }^{4}$ Department of Chemistry, College of William \& Mary, USA \\ ${ }^{\dagger}$ These authors contributed equally to this work
}

\begin{abstract}
A novel selective surface functionalization of 3D micro-silicon channels using a $\mathrm{SiO}_{2}$ nanoparticles (NPs) coating deposited using Layer-by-Layer (LbL) self-assembly technique is reported here. Additionally, application of $\mathrm{SiO}_{2} \mathrm{NPs}$ coating as a stationary phase for chromatographic separation is also presented for the first time. Compared to previously reported techniques, this method enables a simple, low-cost and parallel processing scheme in obtaining homogenous and stable NPs coatings inside the microfluidic channel. Moreover, the reported scheme also provides good repeatability and ease of control over the coating thickness. The $\mathrm{SiO}_{2} \mathrm{NPs}$ functionalized microfabricated gas chromatography columns with wide single capillary channels (1 m-long, 150 m-wide, 240 m-deep) and very narrow multicapillary channels (25 cm-long, $30 \mathrm{~m}$-wide, $240 \mathrm{~m}$-deep, 16 parallel channels) successfully separated a multicomponent gas mixture with a wide range of boiling points.
\end{abstract}

\section{INTRODUCTION}

\section{Microgas Chromatography ( $\mu \mathrm{GC})$}

Gas chromatography (GC) is a reliable chemical analysis technique that is used to separate and identify the constituents of complex gas mixtures. GC has applications in vast areas such as environment monitoring, food processing, pharmaceutical industry, biomedical science, forensic and trace analysis. A chromatographic process (schematically shown in Fig. 1) starts with the injection and vaporization of an unknown sample mixture (analytes); after which the analytes traverse through the separation column via a carrier gas (mobile phase). Columns are typically coated with a polymer (stationary phase) to physically separate the constituents of a mixture into separate compounds in a time domain. Afterwards, the separated components are eluted into a detector that generates a chromatogram based on the retention time of each compound. Each

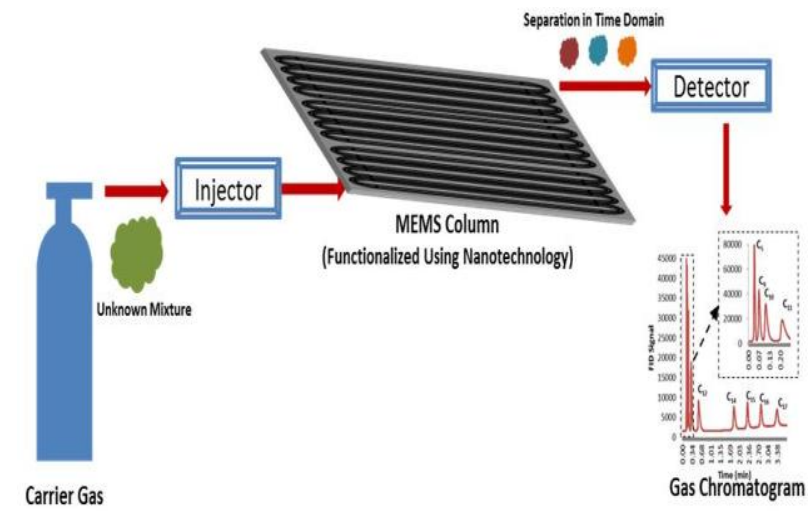

Figure 1. Schematic of a GC system peak in a given chromatogram represents a particular compound.

Traditional bench-top GC instruments, though widely used for over 50 years, are bulky, expensive, time and energy consuming. Due to recent advancement and emergence of microelectromechanical systems (MEMS), energy-efficient integrated $\mu \mathrm{GC}$ systems have attracted considerable attention because of its portability, fast analysis time, and low cost [1-3]. This system, upon complete realization, could enormously expand the range of GC applications in in-situ air quality monitoring, biomedical analysis, and homeland security. As a key component in a $\mu \mathrm{GC}$ system, conventional meters-long separation columns are replaced with coin-sized microfabricated silicon columns. The separation ability and efficiency of the column is directly related to the quality of the stationary phase. Using conventional coating techniques for rectangular-shaped microfabricated columns present major challenges towards getting reproducible and uniform coating. Conventional coating techniques also suffer from limitation in high temperature applications and poor stability (organic coatings) or requirements of expensive instruments and elaborate operation (inorganic coatings) [4-7]. The high thermal stability and high surface area of $\mathrm{SiO}_{2} \mathrm{NPs}$ present an excellent candidate for the stationary phase if employed using simple and low cost methods.

\section{Layer-by-Layer (LbL) Self-Assembly Technique}

Developed first in 1991[8], the LbL self-assembly technique was utilized to develop Ionic Self-Assembly Multilayers (ISAM), which have been widely used in electromechanical actuators [9] as well as in electro-optical and electrochromic devices $[10,11]$. In short, by alternatively dipping the substrate into two aqueous solutions of materials with opposite charges (positive and negative), LbL thin films of the two materials, bonded together by electrostatic force, will build up on each other (two adjacent layers of those two materials are called one bilayer). Thorough rinsing in between each deposition step washes away excess coating materials from the previous stage while leaving enough surface charge for the material to be electrostatically deposited in the next step. Thus, uniform, dense, and sturdy stacks of layers can be deposited on the surface of the substrate with a wide variety of configurations. Additionally, a desired film thickness can be achieved by simply terminating the procedure at a certain number of bilayers. Therefore, with a relatively simple concepts and straightforward operations, the LbL self-assembly technique could be conveniently incorporated into the manufacturing of silicon-based GC devices. In this article, by utilizing the $\mathrm{LbL}$ technique for the first time, high-aspect-ratio (HAR) $\mu \mathrm{GC}$ columns with different configurations are functionalized with $\mathrm{SiO}_{2}$ NPs coatings used as a stationary phase.

\section{FABRICATION}

The fabrication scheme incorporating LbL self-assembled $\mathrm{SiO}_{2}$ NPs coating, schematically shown in Fig. 2, can be divided into three major steps, which include column fabrication, LBL 
deposition of NPs, and anodic bonding of the silicon substrate with Pyrex followed by deactivation of the silica surface.

\section{Column Fabrication}

The fabrication of HAR $1 \mathrm{~m}$-long, 150 m-wide, 240 m-deep single capillary columns ( SCC) and $25 \mathrm{~cm}$-long, $30 \mathrm{~m}$-wide, 240 m-deep multicapillary columns ( MCC) with an array of 16 channels (Fig. 3H) working in parallel starts with anisotropic etching of plain silicon wafers. In the first step, wafers are spin coated with AZ9260 photoresist at $3000 \mathrm{rpm}$, soft-baked at $110^{\circ} \mathrm{C}$ for 1 minute and exposed for 45 seconds using a mask aligner (MA-6). After development using AZ400K, wafers are hard-baked at $110^{\circ} \mathrm{C}$ for 2 minutes. Deep reactive ion etching (DRIE) with photoresist as an etch mask is used to etch the wafers in order to get the desired channel dimensions. Afterwards, the wafer is diced into individual devices. Selective deposition of NPs inside the channels is achieved by using a liftoff procedure explained in the next section (Step 1, Fig. 2).

\section{LbL Deposition of $\mathrm{SiO}_{2} \mathrm{NPs}$}

Keeping the photoresist intact, the $\mathrm{LbL}$ deposition of $\mathrm{SiO}_{2} \mathrm{NPs}$ inside HAR microfluidic channels starts with alternatively dipping each device into a positively charged long-chain inert polymeric aqueous solution (10 mM polyallylamine hydrochloride, $\mathrm{PAH}$, from Sigma-Aldrich), and negatively charged $\mathrm{SiO}_{2} \mathrm{NPs}$ suspension (Nissan Chemical, $45 \mathrm{~nm}$ average particle size). Because of the much narrower channels, the MCC column deposition uses the $\mathrm{SiO}_{2}$ NPs suspension at $1 / 3$ of its original concentration. This prevents the formation of bulky NPs structures between the channel walls. Following our earlier reported findings [11] to guarantee excellent $\mathrm{PAH} / \mathrm{SiO}_{2} \mathrm{NPs}$ LbL coating uniformity, the $\mathrm{pH}$ value of the PAH solution and $\mathrm{SiO}_{2} \mathrm{NPs}$ suspension are adjusted to the values of $7.0( \pm 0.1)$ and $9.0( \pm 0.1)$, respectively, by adding $\mathrm{HCl}$ and $\mathrm{NaOH}$ solutions. The LbL coating is then performed in an automatic dipping system (StratoSequence VI Robot, nanoStrata Inc.) in which every 2.5 minutes of $\mathrm{PAH}$ and $\mathrm{SiO}_{2} \mathrm{NPs}$ coating step is followed by three 1 minute DI water rinsing steps (Step 2, Fig. 2). The LbL deposition scheme presented here affords a homogenous $\mathrm{SiO}_{2}$ NPs coverage both on the interior microchannel surfaces and on the photoresist at the top of chip. After coating, the samples are rinsed thoroughly with deionized (DI) water and dried with low flux nitrogen. NPs coated devices are then dipped in acetone and sonicated for 12 minutes. This liftoff procedure removes the NPs deposited on photoresist without removing the coating inside the trenches. The yield of lift-off process is important especially for the microfluidic applications in which the NPs coated silicon channels are to be sealed with a glass substrate (Step 3, Fig. 2).

\section{Anodic Bonding and Deactivation of the Silica Surface}

Before anodic bonding, the devices are first calcinated in a furnace at a high temperature $\left(500^{\circ} \mathrm{C}\right)$ for 4 hours to fuse the $\mathrm{SiO}_{2}$ NPs together and to ensure the stability of the coating during temperature programming of the $\mathrm{GC}$ columns. Afterwards, the silicon trenches are sealed to a Pyrex wafer using anodic bonding at $400^{\circ} \mathrm{C}$ and $1250 \mathrm{~V}$ (Step 4, Fig. 2). Next, deactivated capillary tubes (220 m outer diameter, $100 \mathrm{~m}$ internal diameter, $25 \mathrm{~cm}$ length) are installed at the inlet and outlet ports using epoxy. Then, $10 \mathrm{mM}$ chlorodimethyl octadecyl silane (CDOS) diluted in toluene is pumped into the chip by nitrogen (Step 5, Fig. 2). This overnight process deactivates NPs and the Pyrex cover surface [12]. Finally, the solvent is blown out by nitrogen and the device is ready for chromatographic analysis.

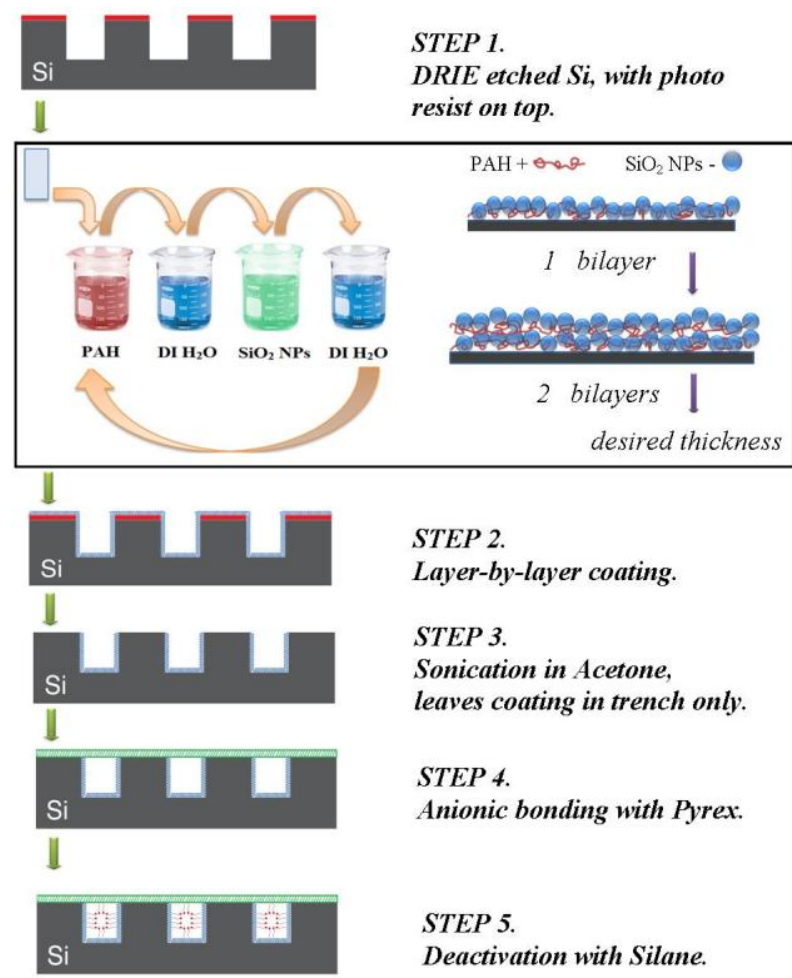

Figure 2. Schematic process flow of $\mathrm{SiO}_{2} \mathrm{NPs}$ coating using Layer-by-Layer self-assembly technique in the Si trench.

\section{CHARACTERIZATION OF $\mathrm{SiO}_{2}$ NPS COATING}

As displayed in Fig. 3 (A-D), the bottom and sidewalls of the trenches are completely covered by $\mathrm{SiO}_{2} \mathrm{NPs}$, while at the same time NPs are neatly removed by the lift-off process from the top surface. This is the first successful demonstration of combining microlithography and LbL self-assembly to selectively deposit NPs on the interior surfaces of silicon microchannels. Fig. 3E and Fig. $3 \mathrm{~F}$ show the SEM images of 5 and 10 bilayers of $\mathrm{SiO}_{2} \mathrm{NPs}$ deposited inside silicon trenches, respectively. The thickness measured directly from the SEM images are $261 \mathrm{~nm}$ to $316 \mathrm{~nm}, 399$ $\mathrm{nm}$ to $486 \mathrm{~nm}$, and $554 \mathrm{~nm}$ to $667 \mathrm{~nm}$ for 5, 10, and 15 bilayers, respectively. Although a lower concentration of $\mathrm{SiO}_{2} \mathrm{NPs}$ suspension is used for MCC, the 10 bilayers coating inside its channel (Fig. 2G) has approximately the same thickness (396 nm) as the 10 bilayers coating inside the SCC (Fig. $2 \mathrm{H})$. This means the coating for the $\mathrm{SiO}_{2}$ NPs suspension at $1 / 3$ of its original concentration is sufficient to deposit a layer of $\mathrm{SiO}_{2} \mathrm{NPs}$ over the previous $\mathrm{PAH}$ layer to neutralize and reverse the surface charge, while the excess $\mathrm{SiO}_{2}$ NPs during the SCC coating with higher concentration suspension is washed off during the rinsing steps. 

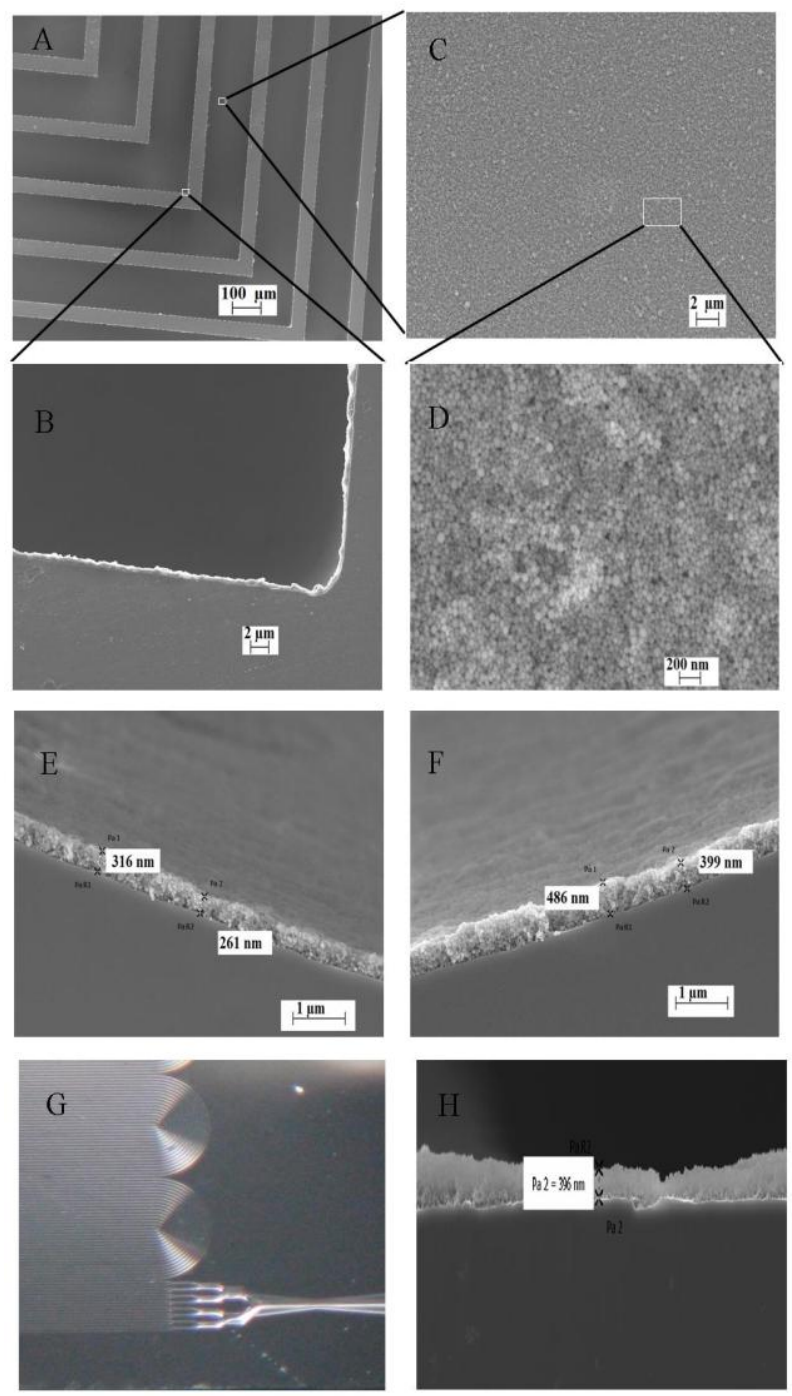

Figure 3. SEM images of the $10 \mathrm{BLs} \mathrm{SiO}_{2} \mathrm{NPs}$ coated single capillary column $(A-D)$, on the side wall $(B)$, bottom of the channel (C-D); cross-section view of silicon trench with $5 \mathrm{BLS}$ (E) and $10 \mathrm{BLs}$ of $\mathrm{SiO}_{2} \mathrm{NPs}$ coating $(F)$. Optical image of multicapillary column $(G)$ and the SEM image of its cross-section with $10 \mathrm{BLs} \mathrm{SiO}_{2} \mathrm{NPs}$ coating on the side wall of Si trench $(H)$.

\section{SEPERATION TEST AND RESULTS}

\section{Experimental Setup}

A commercial GC system (HP 5890 Series II), equipped with an autosampler, electronic pressure controller, and flame ionization detector (FID), was used for characterizing the separation ability of the GC columns with the $\mathrm{SiO}_{2}$ NPs stationary phase. Highly purified nitrogen (from Airgas Inc.) was used as carrier gas. Inlet and detector temperatures are set to $280^{\circ} \mathrm{C}$ and the split ratio is set to 100:1. Standard chemicals were purchased from Sigma-Aldrich and used for all experiments.

\section{Separation Results}

In order to evaluate the chromatographic performance, the functionalized GC columns were placed in a GC oven. All columns were first purged with dry nitrogen to remove any trapped
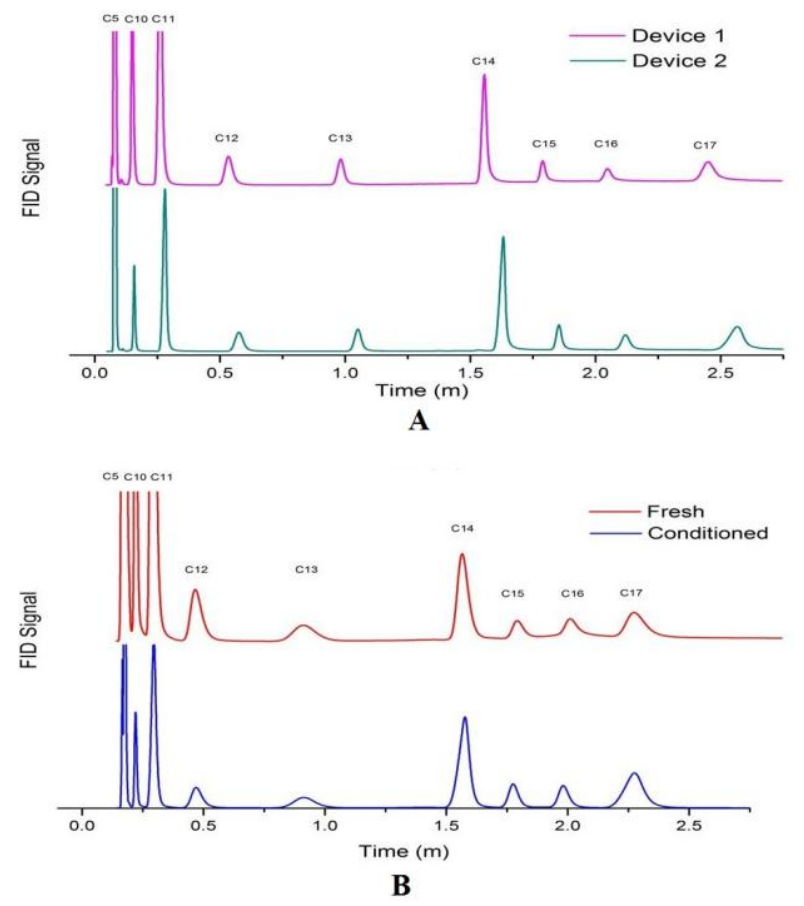

Figure 4. Example of 9 alkane compounds separation by two $10 \mathrm{BLs} \mathrm{SiO}_{2} \mathrm{NPs}$ coated MEMS GC columns (A) and one column before and after 24 hours conditioning under 7.5 psi at $160{ }^{\circ} \mathrm{C}(B)$.

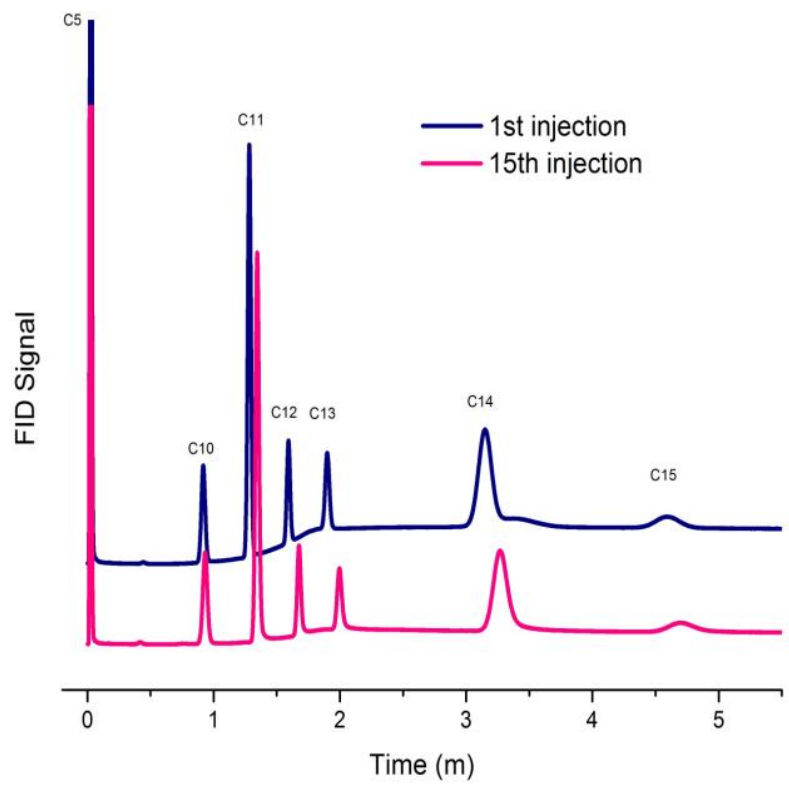

Figure 5. Chromatographic separation result by a $10 \mathrm{BLs} \mathrm{SiO}_{2}$ NPs coated MEMS GC multicapillary column for the 1st and 15 th injection.

oxygen in the system. The columns were then conditioned gradually from ambient temperature to slightly above the maximum 
operating temperature $\left(120^{\circ} \mathrm{C}\right)$ at a temperature ramp rate of $1^{\circ} \mathrm{C} / \mathrm{min}$ and a constant carrier gas flow of 7.5 psi until a constant baseline was observed.

The effectiveness of the $\mathrm{SiO}_{2} \mathrm{NPs}$ coating as a stationary phase for different channel geometries was tested on both SCCs and MCCs. All the chromatograms in Fig. 4 ( SCC) and Fig. 5 ( MCC) exhibited successful separations of alkanes (n-pentane, n-decane, n-undecane, n-dodecane, n-tridecane, n-tetradecane, n-pentadecane, $n$-hexadecane and n-heptadecane) spanning a wide range of boiling points $\left(32^{\circ} \mathrm{C} \sim 202^{\circ} \mathrm{C}\right)$ with high stability and repeatability (less than $4 \%$ deviation in retention time). Fig. 4B shows the long-term stability of this novel stationary phase after $\mathrm{SiO}_{2}$ NPs coated GC columns were subjected to the 7.5 psi flow and $160^{\circ} \mathrm{C}$ temperature for 24 hours. Similarly, Fig. 5 demonstrates the time stability of NPs-based stationary phase after 15 injections on the MCC.

\section{CONCLUSION}

$\mathrm{SiO}_{2}$ NPs coating fabricated by the LbL technique has been incorporated into GC separation columns and utilized as a new stationary phase for the first time. With simple and parallel fabrication procedures, $\mathrm{LbL}$ technique has shown the ability to generate homogenous conformal $\mathrm{SiO}_{2} \mathrm{NPs}$ coating with easy nanoscale thickness control inside the GC columns with different configurations. GC columns (both single and multi- capillary) with this stationary phase provided good separations of an alkane mixture with a wide range of boiling points.

\section{ACKNOWLEDGEMENT}

This work is supported primarily by the National Science Foundation under Award No ECCS-1002279. The authors also want to thank Transducer Research Foundation for generously providing travel support.

\section{REFERENCES}

[1] M. A. Zareian-Jahromi, M. Ashraf-Khorassani, L. T. Taylor, and M. Agah, "Design, Modeling, and Fabrication of MEMS-Based Multicapillary Gas Chromatographic Columns," Journal of Microelectromechanical Systems, 18, 28 (2009).

[2] H. Shakeel, G. Rice, and M. Agah, "First Reconfigurable MEMS Separation Columns for Micro Gas Chromatography," 2012 IEEE 25th International Conference on Micro Electro Mechanical Systems (MEMS), Paris, France, 2012, pp. 823-826.

[3] H. Shakeel and M. Agah, "High-Performance Multicapillary Gas Separation Columns with MPG Stationary Phases," IEEE Sensors, Limerick, Irland, 2011, pp. 1909-1912.
[4] S. Reidy, G. Lambertus, J. Reece, and R. Sacks, "High-Performance, Static-Coated Silicon Microfabricated Columns for Gas Chromatography," Analytical Chemistry, 78, 2623 (2006).

[5] A. Malainou, "The Fabrication of a Microcolumn for Gas Separation Using Poly(dimethylsiloxane) as the Structural and Functional Material," Journal of Micromechanics and Microengineering, 18, 105007 (2008).

[6] L.-M. Yuan, C.-X. Ren, LiLi, P. Ai, Z.-H. Yan, M. Zi, and Z.-Y. Li, "Single-Walled Carbon Nanotubes Used as Stationary Phase in GC," Analytical Chemistry, 78, 6384 (2006).

[7] J. Vial, D. Thiébaut, F. Marty, P. Guibal, R. Haudebourg, K. Nachef, K. Danaie, and B. Bourlon, "Silica Sputtering as a Novel Collective Stationary Phase Deposition for Microelectromechanical System Gas Chromatography column: Feasibility and First Separations," Journal of Chromatography A, 1218, 3262 (2011).

[8] G. Decher, J. D. Hong, and J. Schmitt, "Buildup of Ultrathin Multilayer Films by a Self-Assembly Process, 1 Consecutively Adsorption of Anionic and Cationic Bipolar Amphiphiles on Charged Surfaces," Makromolekulare Chemie, Macromolecular Symposia, 46, 321 (1991).

[9] R. Montazami, S. Liu, Y. Liu, D. Wang, Q. Zhang, and J. R. Heflin, "Thickness Dependence of Curvature, Strain, and Response Time in Ionic Electroactive Polymer Actuators Fabricated via Layer-by-Layer Assembly," Journal of Applied Physics, 109, 104301 (2011).

[10] R. Montazami, V. Jain, and J. R. Heflin, "High Contrast Asymmetric Solid State Electrochromic Devices Based on Layer-by-Layer Deposition of Polyaniline and Poly(aniline sulfonic Acid)," Electrochimica Acta, 56, 990 (2010).

[11] S. E. Yancey, W. Zhong, J. R. Heflin, and A. L. Ritter, "The Influence of Void Space on Antireflection Coatings of Silica Nanoparticle Self-Assembled Films," Journal of Applied Physics, 99, 034313 (2006).

[12] A. D. Radadia, R. I. Masel, M. A. Shannon, J. P. Jerrell, and K. R. Cadwallader, "Micromachined GC Columns for Fast Separation of Organophosphonate and Organosulfur Compounds," Analytical Chemistry, 80, 4087 (2008).

CONTACT

*Dr. M. Agah, tel: +1-540-231-2653; Agah@VT.edu 